J. Austral. Math. Soc. 21 (Series A) (1976), 96-107.

\title{
A FUNCTIONAL CALCULUS FOR SESQUIHERMITIAN OPERATORS ON QUATERNIONIC HILBERT SPACE
}

\author{
N. C. POWERS
}

(Received 2 December 1974)

\begin{abstract}
A continuous real-linear operator $A=A_{0}+i_{1} A_{1}+i_{2} A_{2}+i_{3} A_{3}$ on a quaternionic Hilbert space is called sesquihermitian if the linear operators $A_{v}$ are Hermitian; this condition is independent of the choice of quaternion basis $\left(i_{1}, i_{2}, i_{3}\right)$. The joint spectral distribution of the $A_{\nu}$ provides a functional calculus for sesquihermitian operators and real-valued $C^{\infty}$-functions on 2 . This calculus is independent of the quaternion basis and extends naturally to quaternion-valued functions to give a continuous quaternion-linear mapping from the algebra of these functions to that of sesquihermitian operators. The mapping is not, in general, multiplicative unless the $A_{\nu}$ commute, in which case it agrees with that for several commuting operators on complex Hilbert space.

The convex hull of the support of the joint spectral distribution coincides with the closure of the numerical range of $A$ and contains the spectrum of $A$.
\end{abstract}

\section{Introduction}

Let $A$ be a continuous real-linear operator on a quaternionic Hilbert space $\mathscr{H}$ and $\left(i_{1}, i_{2}, i_{3}\right)$ be a quaternion basis (i.e., a right-handed orthonormal triad of vector quaternions). Powers (1973) has shown that $A$ has a canonical decomposition $A=\sum_{v=0}^{3} i_{v} A_{\nu}$, where $i_{0}=1$ and where $A_{0}, A_{1}, A_{2}, A_{3}$ are continuous (quaternion-) linear operators uniquely determined by the choice of quaternion basis. In the present work a functional calculus is developed for a class of real-linear operators which we call sesquihermitian. This calculus is based on the Weyl functional calculus developed by Anderson $(1967,1969,1970)$ for (not necessarily commuting) $n$-tuples of self-adjoint operators on a (complex) Banach space.

The research for this paper was carried out at the Flinders University of South Australia with the support of a Commonwealth Postgraduate award and subsequently of a Flinders University Research Scholarship. 
Further information and references on quaternionic Hilbert space can be found in Powers (1973).

The author wishes to thank his Ph.D. supervisor, Professor B. Abrahamson of the Flinders University of South Australia, for his advice and encouragement while this research was being carried out.

\section{Sesquihermitian operators}

The adjoint $A^{*}$ of a continuous real-linear operator $A$ on $\mathscr{H}$ has been defined in Powers (1973) thus

$$
\operatorname{Re}\langle A x, y\rangle=\operatorname{Re}\left\langle x, A^{*} y\right\rangle \text { for each } x, y \in \mathscr{H} .
$$

If $A$ has canonical decomposition $\sum_{v=0}^{3} i_{\nu} A_{\nu}$, then

$$
A^{*}=A_{0}^{*}-i_{1} A_{1}{ }^{*}-i_{2} A_{2}{ }^{*}-i_{3} A_{3}{ }^{*} .
$$

4 is said to be Hermitian if $A^{*}=A$, normal if $A A^{*}=A^{*} A$.

In the case where the linear operators $A_{0}, A_{1}, A_{2}, A_{3}$ are Hermitian, the Weyl functional calculus can be applied to the quadruple $\left(A_{0}, A_{1}, A_{2}, A_{3}\right)$. First, lowever, we show that this condition is independent of the choice of juaternion basis.

DEFINITION. Let $\sum_{v=0}^{3} i_{v} A_{\nu}$ be the canonical decomposition of a continuous -eal-linear operator $A$ on a quaternionic Hilbert space $\mathscr{H}$. The operator $A-A_{0}$ $s$ called the vector part $A_{v}$ of $A$. If $A_{0}=0$, then $A$ is called a vector operator. The operator $A$ is called sesquihermitian if $A_{0}$ is Hermitian and $A_{v}$ is ikew-Hermitian.

Powers (1973) showed that $A_{0}$ is independent of the quaternion basis $\left.i_{1}, i_{2}, i_{3}\right)$. Hence the above definitions are also basis-free.

Theorem 1. Let $A$ be a continuous real-linear operator on $\mathscr{H}$ with anonical decomposition $\sum_{v=0}^{3} i_{\nu} A_{\nu}$. Then $A$ is sesquihermitian if and only if the $\mathbf{f}_{\boldsymbol{v}}$ are Hermitian.

Proof. If $A_{1}, A_{2}$ and $A_{3}$ are Hermitian, then the operator $A_{v}=\sum_{v=1}^{3} i_{\nu} A_{v}$ is kew-Hermitian. If $A_{0}$ is also Hermitian, then $A$ is sesquihermitian.

Conversely, suppose that $A$ is sesquihermitian. Then $A_{0}$ is Hermitian and $t_{v}$ is skew-Hermitian. Applying the canonical decomposition [Theorem 2 of 'owers (1973)] to $A_{\mathrm{v}}$, we have:

$$
\begin{aligned}
& 4 A_{1}=-i_{1} A_{v}-A_{v} \cdot i_{1} I-i_{3} A_{v} \cdot i_{2} I+i_{2} A_{v} \cdot i_{3} I, \\
& 4 A_{2}=-i_{2} A_{v}+i_{3} A_{v} \cdot i_{1} I-A_{v} \cdot i_{2} I-i_{1} A_{v} \cdot i_{3} I, \\
& 4 A_{3}=-i_{3} A_{v}-i_{2} A_{v} \cdot i_{1} I+i_{1} A_{v} \cdot i_{2} I-A_{v} \cdot i_{3} I .
\end{aligned}
$$


It can now be verified that $A_{1}, A_{2}$, and $A_{3}$ are Hermitian by direct computation, using the fact that $\left(i_{\nu} I\right)^{*}=-i_{\nu} A(\nu=1,2,3)$.

\section{The joint spectral distribution}

Throughout the rest of this work $A$ denotes a sesquihermitian operator on $\mathscr{H}$ with canonical decomposition $\Sigma_{\nu=0}^{3} i_{v} A_{\nu}$. Given an arbitrary unit vector quaternion $u, \mathscr{H}_{1}(u)$ denotes the symplectic image of $u$ as defined in Powers (1973). Then $\left(A_{0}, A_{1}, A_{2}, A_{3}\right)$ is a quadruple of Hermitian operators on the complex Hilbert space $\mathscr{H}_{1}(u)$ and thus has a joint spectral distribution in the sense of Anderson (1969). For the time being we denote this distribution by $T_{u}\left(A_{0}, A_{1}, A_{2}, A_{3}\right)$; however, it will turn out to be independent both of $u$ and of the quaternion basis $\left(i_{1}, i_{2}, i_{3}\right)$.

Given a quaternion $q=\Sigma_{v=0}^{3} q_{\nu} i_{\nu}$, let $q$ denote the real 4-vector $\left(q_{0}, q_{1}, q_{2}, q_{3}\right)$ and let $d q$ denote integration with respect to the four real variables. The Hermitian linear operator $\Sigma_{v=0}^{3} q_{v} A_{\nu}$ is denoted by $q \cdot A$. Let $C^{\infty}\left(R^{4}\right)$ denote the class of those real-valued functions $f=f(q)$ which are infinitely differentiable with respect to the four real variables $\left(q_{0}, q_{1}, q_{2}, q_{3}\right)$ and let $\mathscr{D}\left(\boldsymbol{R}^{4}\right)$ consist of those members of $C^{\infty}\left(\boldsymbol{R}^{4}\right)$ which have compact support.

Definition. Any function $f: 2 \rightarrow 2$ can be written in the form:

$$
f(q)=\sum_{\nu=0}^{3} i_{\nu} f_{\nu}(q) \text { for each } q \in \mathscr{Q} .
$$

where the $f_{v}$ are real-valued functions. The function $f$ is called a $C^{\infty}$-function if each $f_{v} \in C^{\infty}\left(\boldsymbol{R}^{4}\right)$.

THEOrem 2. Let $\left(i_{1}, i_{2}, i_{3}\right)$ and $\left(i_{1},{ }^{\prime}, i_{2}{ }^{\prime}, i_{3}{ }^{\prime}\right)$ be two quaternion bases and let $A$ be the sesquihermitian operator on $\mathscr{H}$ given by

$$
\left.A=\sum_{\nu=0}^{3} i_{\nu} A_{v}=\sum_{v=0}^{3} i_{\nu}{ }^{\prime} A_{\nu}{ }^{\prime} . \quad \text { (writing } i_{0}=i_{0}{ }^{\prime}=1\right) .
$$

Let $f: \mathscr{Q} \rightarrow \boldsymbol{R}$ be a $C^{\infty}$-function and write:

$$
f(q)=g\left(q_{0}, q_{1}, q_{2}, q_{3}\right)=h\left(q_{0_{0}}, q_{1}{ }^{\prime}, q_{2}{ }^{\prime}, q_{3}{ }^{\prime}\right),
$$

where $q=\sum_{v=0}^{3} q_{v} i_{v}=\sum_{v=0}^{3} q_{v}{ }^{\prime} i_{v}{ }^{\prime}$. Then

$$
T_{u}\left(A_{0}, A_{1}, A_{2}, A_{3}\right) g=T_{u}\left(A_{0}{ }^{\prime}, A_{1}{ }^{\prime}, A_{2}{ }^{\prime}, A_{3}{ }^{\prime}\right) h .
$$

Proof. Let $L$ be the $3 \times 3$ real orthogonal matrix such that

$$
\left[i_{1}{ }^{\prime} i_{2}{ }^{\prime} i_{3}{ }^{\prime}\right]=\left[i_{1} i_{2} i_{3}\right] L \text {, }
$$

and let $M$ be the $4 \times 4$ matrix $\left[\begin{array}{c:c}1 & 0 \\ \hdashline 0 & L\end{array}\right]$.

Then $\left[q_{0}{ }^{\prime} q_{1}^{\prime} q_{2}^{\prime} q_{3}^{\prime}\right]=\left[q_{0} q_{1} q_{2} q_{3}\right] M$, and [Powers (1973) Theorem 3]:

$$
\left[A_{0}{ }^{\prime} A_{1}{ }^{\prime} A_{2}{ }^{\prime} A_{3}{ }^{\prime}\right]=\left[A_{0} A_{1} A_{2} A_{3}\right] M .
$$


Anderson [(1969), Theorem 2.9 (a)] has proved that, if $B=\left(B_{1} ; \cdots, B_{n}\right)$ is an $n$-tuple of Hermitian operators on a complex Hilbert space and $S$ is an affine transformation of $\boldsymbol{R}^{n}$, then $T(S B) f=T(\boldsymbol{B})(S f)$ for each $f \in C^{\infty}\left(\boldsymbol{R}^{n}\right)$, where $S f$ is the function on $\boldsymbol{R}^{n}$ defined by $(S f)(\boldsymbol{x})=f(S \boldsymbol{x})$ for each $\boldsymbol{x} \in \boldsymbol{R}^{n}$, and $S \boldsymbol{B}$ is the $n$-tuple of operators obtained by replacing the components of $x$ in the expansion of $S x$ by the operators $B_{1}, \cdots, B_{n}$. Applying this result to the affine transformation $M$, the function $h$, and the quadruple $\left(A_{0}, A_{1}, A_{2}, A_{3}\right)$, we have:

$$
T_{u}\left(A_{0}{ }^{\prime}, A_{1}{ }^{\prime}, A_{2}{ }^{\prime}, A_{3}{ }^{\prime}\right) h=T_{u}\left(A_{0}, A_{1}, A_{2}, A_{3}\right)(h \circ M) \text {. But } h \circ M=g,
$$

so the theorem is proved.

Thus, for any $C^{\infty}$-function $f: \mathscr{Q} \rightarrow R$, we can write $T_{u}(A) f$ instead of $T_{u}\left(A_{0}, A_{1}, A_{2}, A_{3}\right) f$ without ambiguity.

THEOREM 3. For each sesquihermitian operator $A$ on $\mathscr{H}$ and each $C^{\infty}$ function $f: \mathscr{Q} \rightarrow \boldsymbol{R}, T_{u}(A) f$ is a Hermitian linear operator and is independent of u.

Proof. Since $\mathscr{D}\left(\boldsymbol{R}^{4}\right)$ is dense in $C^{\infty}\left(\boldsymbol{R}^{4}\right)$ and $T_{u}(A)$ is continuous on $C^{\infty}\left(\boldsymbol{R}^{4}\right)$ [Anderson (1969), Theorem 2.9 (c)], it is sufficient to prove the assertion for $f \in \mathscr{D}\left(\boldsymbol{R}^{4}\right)$.

Let $f \in \mathscr{D}\left(\boldsymbol{R}^{4}\right)$. Then, by definition of $T_{u}(A)$,

$$
\begin{aligned}
& T_{u}(A) f=(2 \pi)^{-2} \int_{\mathbb{R}^{4}} \hat{f}_{u}(q) \exp (-u q \cdot \boldsymbol{A}) d q, \\
& \text { where } \hat{f}_{u}(q)=(2 \pi)^{-2} \int_{\boldsymbol{R}^{4}} f(p) \exp (u p \cdot q) d \boldsymbol{p}
\end{aligned}
$$

for each $q \in 2$. The operator $\exp (-u q \cdot A)$ is given by the usual functional calculus for a single operator on the complex Hilbert space $\mathscr{H}_{1}(u)$. It can be written in the form:

$$
\exp (-u \boldsymbol{q} \cdot \boldsymbol{A})=\cos (\boldsymbol{q} \cdot \boldsymbol{A})-u \sin (\boldsymbol{q} \cdot \boldsymbol{A}) .
$$

The operator $\cos (\boldsymbol{q} \cdot \boldsymbol{A})$ and $\sin (\boldsymbol{q} \cdot \boldsymbol{A})$ can be expressed as the strong limits of the usual power series; since these two series have real co-efficients, $\cos (\boldsymbol{q} \cdot \boldsymbol{A})$ and $\sin (\boldsymbol{q} \cdot \boldsymbol{A})$ are (quaternion-) linear operators. Thus we have:

$$
T_{u}(A) f=(2 \pi)^{-4} \int_{R^{4}} \int_{R^{4}} f(p) \exp (u p \cdot q) \exp (-u q \cdot A) d p d q
$$

$$
=f_{0}(A)+u f_{1}(A),
$$

where $f_{0}(A)$ and $f_{1}(A)$ are (quaternion-) linear operators given by:

$$
f_{0}(A)=(2 \pi)^{-4} \int_{R^{4}} \int_{R^{4}} f(p)[\cos (p \cdot q) \cos (q \cdot A)+\sin (p \cdot q) \sin (q \cdot A)] d p d q
$$


and

$$
f_{1}(A)=(2 \pi)^{-4} \int_{R^{4}} \int_{R^{4}} f(p)[\sin (p \cdot q) \cos (q \cdot A)-\cos (p \cdot q) \sin (q \cdot A)] d p d q .
$$

If we replace $u$ by $-u$ in (2) we obtain:

$$
\begin{aligned}
T_{-u}(A) f & =f_{0}(A)-u f_{1}(A) \\
& =(2 \pi)^{-4} \int_{\boldsymbol{R}^{4}} \int_{\boldsymbol{R}^{4}} f(p) \exp (-u p \cdot q) \exp (u q \cdot \boldsymbol{A}) d p d q \\
& =(2 \pi)^{-4} \int_{\boldsymbol{R}^{4}} \int_{\boldsymbol{R}^{4}} f(p) \exp \left(u p \cdot q^{\prime}\right) \exp \left(-u q^{\prime} \cdot \boldsymbol{A}\right) d p d q^{\prime},
\end{aligned}
$$

putting $\boldsymbol{q}^{\prime}=-\boldsymbol{q}$ and nothing that the Jacobian of $\boldsymbol{q}^{\prime}$ with respect to $\boldsymbol{q}$ is +1 . Thus $T_{-u}(A) f=T_{u}(A) f$. Hence $f_{1}(A)=0$, so that $T_{u}(A) f=f_{0}(A)$. Hence $T_{u}(A) f$ is linear and independent of $u$. Anderson [(1969), Theorem $\left.2.9(\mathrm{~d})\right]$ has shown that $T_{u}(A) f$ is Hermitian whenever $f$ is real-valued.

Thus we can write $T(A)$ instead of $T_{u}(A)$ without ambiguity. The support $\sigma_{w}(A)$ of the distribution $T(A)$ is called the joint spectrum of the sesquihermitian operator $A$.

\section{The joint spectrum}

The aim of this section is to investigate the relationship between the joint spectrum, the "traditional" spectrum, and the numerical range of a sesquihermitian operator.

DEFINITION. The numerical range $W(A)$ of a continuous real-linear operator $A$ on a quaternionic Hilbert space is the set $W(A)=$ $\{\langle A x, x\rangle: x \in \mathscr{H},\|x\|=1\}$.

Clearly the numerical range of a Hermitian linear operator is contained in $\boldsymbol{R}$.

THEOREM 4. If $A$ is a sesquihermitian operator on a quaternionic Hilbert space $\mathscr{H}$, then $W(A)$ is convex.

Proof. Let $p=\langle A x, x\rangle, q=\langle A y, y\rangle$, where $\|x\|=\|y\|=1$. We have to show that the segment $\{t p+(1-t) q: 0 \leqq t \leqq 1\}$ lies in $W(A)$.

(i) If $p=q$, the result is trivial. So assume $p \neq q$. Assume further that the theorem has been proved for the case where $p=1, q=0$. In the general case, there exist quaternions $a$ and $b$ such that $a p+b=1, a q+b=0$. Consider the canonical decomposition $\sum_{\nu=0}^{3} i_{\nu}(a A+b I)_{\nu}$ of the operator $a A+b I$. Each of the linear operators $(a A+b I)_{\nu}$ is Hermitian, being a real-linear combination of the Hermitian operators $A_{0}, A_{1}, A_{2}, A_{3}$, and the identity operator $I$. But $\langle(a A+b I) x, x\rangle=1$ and $\langle(a A+b I) y, y\rangle=0$. So, by our assumption that the 
theorem is true for this case, $[0,1] \subseteq W(a A+b I)$. Now let $t \in[0,1]$. Then there is an element $z \in \mathscr{H}$ such that $\|z\|=1$ and:

$$
\langle a A z+b z, z\rangle=a\langle A z, z\rangle+b=t .
$$

But $t=t(a p+b)+(1-t)(a q+b)=a[t p+(1-t) q]+b$. Hence $t p+(1-t) q=\langle A z, z\rangle \in W(A)$. Thus we can assume without loss of generality that $p=1, q=0$.

(ii) Let $\langle A x, x\rangle=1,\langle A y, y\rangle=0$, where $\|x\|=\|y\|=1$. Then

$$
\langle A(u x), u x\rangle=1
$$

where $u$ is an arbitrary unit quaternion. To show this, we note that:

$$
\begin{aligned}
\langle A(u x), u x\rangle & =\sum_{\nu=0}^{3} i_{\nu}\left\langle A_{\nu}(u x), u x\right\rangle \\
& =\sum_{\nu=0}^{3} i_{\nu} u\left\langle A_{\nu} x, x\right\rangle u^{\sigma},
\end{aligned}
$$

where $\boldsymbol{u}^{\sigma}$ denotes the quaternion conjugate of $u$. But, since the $A_{\nu}$ are hermitian, $\langle A, x, x\rangle \in \boldsymbol{R},(\nu=0,1,2,3)$.

Hence $\langle A(u x), u x\rangle=\sum_{\nu=0}^{3} i_{\nu}\left\langle A_{\nu} x, x\right\rangle=\langle A x, x\rangle=1$. Now it is possible to choose a unit quaternion $u$ such that:

$$
\operatorname{Re}\left\langle A_{\nu}(u x), y\right\rangle=0 . \quad(\nu=1,2,3)
$$

To prove this we have to find a unit quaternion $u=\sum_{\wedge=0}^{3} u_{\lambda} i_{\lambda}$ such that

$$
u_{0}\left\langle A_{\nu} x, y\right\rangle_{0}-\sum_{\lambda=1}^{3} u_{\lambda}\left\langle A_{\nu} x, y\right\rangle_{\lambda}=0 \quad(\nu=1,2,3)
$$

where the $\left\langle A_{\nu} x, y\right\rangle_{\lambda}$ are the real components of the quaternions $\left\langle A_{\nu} x, y\right\rangle$. But (5) is a system of three homogeneous linear equations in the four unknowns $u_{0}, u_{1}, u_{2}, u_{3}$ and hence has a non-trivial solution. Thus it is always possible to choose a suitable unit quaternion $u$. that:

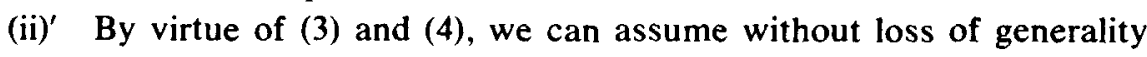

$$
\operatorname{Re}\left\langle A_{\nu} x, y\right\rangle=0 \quad(\nu=1,2,3)
$$

(iii) With the assumptions justified in parts (i) and (ii)', let $z(t)=$ $t x+(1-t) y$ for each $t \in[0,1]$. Then $z(t)$ does not vanish on $[0,1]$. For suppose $z(t)=0$ for some $t \in[0,1]$. Since $\|x\|=\|y\|=1, t \neq 0$ or 1 . So we can write $x=t^{-1}(t-1) y$, contradicting the assumption that $\langle A x, x\rangle=1$ and $\langle A y, y\rangle=0$.

Thus, for each $t \in[0,1]$, it is possible to write $\theta(t)=\|z(t)\|^{-2}\langle A z(t), z(t)\rangle$. Clearly $\theta$ is a continuous function on $[0,1]$ and $\theta(0)=0, \theta(1)=1$. Furthermore, $\theta$ is real-valued. For: 


$$
\begin{array}{rlr}
\left\langle A_{\nu} z(t), z(t)\right\rangle= & t^{2}\left\langle A_{\nu} x, x\right\rangle+t(1-t)\left[\left\langle A_{\nu} x, y\right\rangle+\left\langle A_{\nu} y, x\right\rangle\right] \\
& +(1-t)^{2}\left\langle A_{\nu} y, y\right\rangle \quad(\nu=1,2,3) .
\end{array}
$$

But, by an argument similar to that used in (ii),

$$
\left\langle A_{\nu} x, x\right\rangle=\left\langle A_{\nu} y, y\right\rangle=0 \text {. }
$$

Furthermore, since the $A_{\nu}$ are Hermitian,

$$
\left\langle A_{\nu} x, y\right\rangle+\left\langle A_{\nu} y, x\right\rangle=2 \operatorname{Re}\left\langle A_{\nu} x, y\right\rangle=0 .
$$

Hence $\left\langle A_{v} z(t), z(t)\right\rangle=0(\nu=1,2,3)$ so that $\langle A z(t), z(t)\rangle \in \boldsymbol{R}$ for each $t \in[0,1]$. Thus $\theta$ is a real-valued, continuous function on $[0,1]$ such that $\theta(0)=0, \theta(1)=1$. Hence $[0,1] \subseteq \theta([0,1])$, so that $[0,1] \subseteq W(A)$ as required.

THEOREM 5. If $A$ is a sesquihermitian operator on a quaternionic Hilbert space $\mathscr{H}$, then the convex hull of $\sigma_{w}(A)$ is equal to the closure of the numerical range $W(A)$.

Proof. Let $A$ be a sesquihermitian operator on $\mathscr{H}$. Then, by Theorem 4, $W(A)$ is convex. Hence its closure $[W(A)]^{-}$is also convex. Now Anderson [(1969), Theorem 3.5] has shown that, if $B=\left(B_{1}, \cdots, B_{n}\right)$ is an $n$-tuple of Hermitian operators on a complex Hilbert space and $\sigma_{w}(B)$ is the support of the joint spectral distribution $T(\boldsymbol{B})$, then the convex hull $\operatorname{ch} \sigma_{w}(B)$ of $\sigma_{w}(B)$ is given by:

$$
\operatorname{ch} \sigma_{w}(B)=\bigcap_{|\xi|=1}\left\{\eta \in R^{n}: \inf \xi \cdot B \leqq \xi \cdot \eta \leqq \sup \xi \cdot B\right\}
$$

where $\boldsymbol{\xi} \cdot \boldsymbol{B}=\sum_{i=1}^{n} \xi_{i} B_{i}$ and $\boldsymbol{\xi}$ ranges over $\boldsymbol{R}^{n}$. Applying this result to the quadruple $\left(A_{0}, A_{1}, A_{2}, A_{3}\right)$, we have:

$$
\operatorname{ch} \sigma_{w}(A)=\bigcap_{u \in q}\{q \in 2: \inf u \cdot A \leqq q \cdot u \leqq \sup u \cdot A\}
$$

where $\mathcal{U}$ denotes the set of unit quaternions. Let $q=\sum_{\nu=0}^{3} q_{v} i_{\nu} \in[W(A)]^{-}$. Then $q_{\nu} \in\left[W\left(A_{\nu}\right)\right]^{-}(\nu=0,1,2,3)$ and so $\boldsymbol{u} \cdot \boldsymbol{q} \in[W(\boldsymbol{u} \cdot \boldsymbol{A})]^{-}$for each $\boldsymbol{u} \in \mathcal{U}$. But $\boldsymbol{u} \cdot \boldsymbol{A}$ is a Hermitian linear operator on $\mathscr{H}$, with real numerical range, so we have as a trivial consequence of the corresponding result for complex Hilbert space:

$$
[\boldsymbol{W}(\boldsymbol{u} \cdot \boldsymbol{A})]^{-}=[\inf \boldsymbol{u} \cdot \boldsymbol{A}, \sup \boldsymbol{u} \cdot \boldsymbol{A}] .
$$

Thus inf $\boldsymbol{u} \cdot \boldsymbol{A} \leqq \boldsymbol{u} \cdot \boldsymbol{q} \leqq \sup \boldsymbol{u} \cdot \boldsymbol{A}$ for each $\boldsymbol{q} \in[\boldsymbol{W}(\boldsymbol{A})]^{-}$. Hence $[\boldsymbol{W}(A)]^{-} \subseteq$ ch $\sigma_{w}(A)$. Moreover, given any $u \in \mathcal{U}$, we can find quaternions $p$ and $q$ in $[W(A)]^{-}$such that $\boldsymbol{u} \cdot \boldsymbol{p}=\inf \boldsymbol{u} \cdot \boldsymbol{A}$ and $\boldsymbol{u} \cdot \boldsymbol{q}=\sup \boldsymbol{u} \cdot \boldsymbol{A}$. Thus $[\boldsymbol{W}(A)]^{-}$has the same bounding hyperplanes as $\operatorname{ch} \sigma_{w}(A)$. But $[W(A)]^{-}$is convex, so $[W(A)]^{-}=$ $\operatorname{ch}_{w}(A)$.

We now examine the relationship between the spectrum and the numerical range. 
DefinItion. Let $A: \mathscr{D}(A) \subseteq \mathscr{H} \rightarrow \mathscr{H}$ be a (not necessarily bounded) reallinear operator. The resolvent set $\rho(A)$ is defined to be the set of all quaternions $q$ such that the operator $A-q I$ has a bounded inverse $(A-q I)^{-1}$ defined on all of $\mathscr{H}$. The spectrum $\sigma(A)$ is the complement of $\rho(A)$ in 2 and can be expressed as the union of three disjoint parts in the usual way, viz,:

(i) the point spectrum $\sigma_{P}(A)=\{q \in \mathscr{Q}: A-q I$ is not injective $\}$,

(ii) the continuous spectrum $\sigma_{C}(A)$ consisting of those quaternions $q$ such that $A-q I$ is injective and the range $\mathscr{R}(A-q I)$ is a proper, dense subset $\mathscr{H}$, and:

(iii) the residual spectrum $\sigma_{R}(A)$ consisting of those quaternions q such that $\mathscr{R}(A-q I)$ is injective but $\mathscr{R}(A-q I)$ is not dense in $\mathscr{H}$.

Definition. The approximate point spectrum $\Pi(A)$ of a continuous real-linear operator $A: \mathscr{H} \rightarrow \mathscr{H}$ is the set of quaternions $q$ such that $A-q I$ is not bounded below. The compression spectrum $\Gamma(A)$ is the set of quaternions $q$ such that $\mathscr{R}(A-q I)$ is not dense in $\mathscr{H}$.

As in the complex Hilbert space theory, it is easy to verify that:

(i) $\sigma(A)=\Pi(A) \cup \Gamma(A)$,

(ii) $\sigma_{P}(A) \subseteq \Pi(A)$,

(iii) $\sigma_{R}(A)=\Gamma(A)-\sigma_{P}(A)$,

(iv) $\sigma_{C}(A)=\Pi(A)-\left[\Gamma(A) \cup \sigma_{P}(A)\right]$.

THEOREM 6. If the operator $A: \mathscr{H} \rightarrow \mathscr{H}$ is sesquihermitian, then $\sigma(A) \subseteq[W(A)]^{-}$.

The proof makes use of two lemmas.

Lemma 1. For every continuous real-linear operator $A: \mathscr{H} \rightarrow \mathscr{H}$, $\Pi(A) \subseteq[W(A)]^{-}$.

Proof. Let $q \in \Pi(A)$. Then there is a sequence $\left\{x_{n}\right\}$ in $\mathscr{H}$ such that $\left\|x_{n}\right\|=1$ for all $n$ and $\lim _{n}(A-q I) x_{n}=0$. Hence $\left|\left\langle A x_{n}, x_{n}\right\rangle-q\right|=$ $\left|\left\langle(A-q I) x_{n}, x_{n}\right\rangle\right| \leqq\left\|(A-q I) x_{n}\right\|$ which $\rightarrow 0$ as $n \rightarrow \infty$. So $\lim _{n}\left\langle A x_{n}, x_{n}\right\rangle=q$, so that $q \in[W(A)]^{-}$.

Lemma 2. If $A: \mathscr{H} \rightarrow \mathscr{H}$ is a continuous real-linear operator, then $\sigma_{P}\left(A^{*}\right)=\left\{q^{\sigma}: q \in \Gamma(A)\right\}$.

Proof. $q^{\sigma} \in \sigma_{P}\left(A^{*}\right)$ if and only if there is an element $x \in \mathscr{H}$ such that $\left(A^{*}-q^{\sigma} I\right) x=0$.

But $\left(A^{*}-q^{\sigma} I\right) x=0 \Leftrightarrow \operatorname{Re}\left\langle\left(A^{*}-q^{\sigma} I\right) x, y\right\rangle=\operatorname{Re}\langle x,(A-q I) y\rangle=0$ for each $y \in \mathscr{H}$. Thus $q^{\sigma} \in \sigma_{P}\left(A^{*}\right)$ if and only if $\mathscr{R}(A-q I)$ is not dense in $\mathscr{H}-$ i.e., if and only if $q \in \Gamma(A)$.

Proof of Theorem 6. By Lemma 1 , we need only show that $\Gamma(A) \subseteq$ $[W(A)]^{-}$. Let $q \in \Gamma(A)$. Then, by Lemma 2 , there is an element $x \in \mathscr{H}$ such 
that $A^{*} x-q^{\sigma} x=0$. We can assume without loss of generality that $\|x\|=1$. Then $\left\langle A^{*} x, x\right\rangle=q^{*}$. Now let $\sum_{v=0}^{3} i_{v} A_{\nu}$ be the canonical decomposition of $A$ with respect to a quaternion basis $\left(i_{1}, i_{2}, i_{3}\right)$. Then, since $A$ is sesquihermitian, $A^{*}=A_{0}-\sum_{\nu=1}^{3} i_{\nu} A_{\nu}$. Thus $\left\langle A^{*} x, x\right\rangle=\left\langle A_{0} x, x\right\rangle-\Sigma_{\nu=1}^{3} i_{\nu}\left\langle A_{\nu} x, x\right\rangle=\langle A x, x\rangle^{\sigma}$ since $\left\langle A_{\nu} x, x\right\rangle \in \boldsymbol{R}(\nu=0,1,2,3)$. Thus $\langle A x, x\rangle=\left\langle A^{*} x, x\right\rangle^{\sigma}=q$, so that $q \in W(A)$. Hence $\Gamma(A) \subseteq W(A)$, so that $\sigma(A)=\Pi(A) \cup \Gamma(A) \subseteq[W(A)]^{-}$.

Corollary. If $A$ is sesquihermitian, then $\sigma(A) \subseteq \operatorname{ch} \sigma_{w}(A)$.

The last step of the proof of Theorem 6 breaks down if $A$ is not sesquihermitian; in this case the $\left\langle A_{\nu} x, x\right\rangle$ need not be real and we cannot assert that $\left\langle A^{*} x, x\right\rangle=\langle A x, x\rangle^{\text {. }}$. The truth or falsity of the theorem for the general case is still an open question. However, the proof remains valid in the case where $A$ is linear but not necessarily Hermitian.

The assertion of Theorem 6 can also be proved for an arbitrary normal real-linear operator.

THEOREM 7. The residual spectrum of a continuous normal real-linear operator $A$ on $\mathscr{H}$ is empty.

Proof. Let $A$ be a continuous normal real-linear operator on $\mathscr{H}$ and let $q$ be any quaternion.

Then $\quad(A-q I)\left(A^{*}-q^{\sigma} I\right)-\left(A^{*}-q^{\sigma} I\right)(A-q I)=\left(q^{\sigma} A-A \cdot q^{\sigma} I\right)$ $-\left(q A^{*}-A^{*} \cdot q I\right)$. A routine calculation involving the canonical decompositions of $A$ and $A^{*}$ shows the operator on the right-hand side is zero, so that the operator $A_{q}=A-q I$ is normal. Thus for each $x \in \mathscr{H}$ :

$$
\begin{aligned}
\left\|\left(A_{q}\right)^{*} x\right\|^{2} & =\left\langle\left(A_{q}\right)^{*} x,\left(A_{q}\right)^{*} x\right\rangle=\operatorname{Re}\left\langle A_{q}\left(A_{q}\right)^{*} x, x\right\rangle \\
& =\operatorname{Re}\left\langle\left(A_{q}\right)^{*} A_{q} x, x\right\rangle=\left\langle A_{q} x, A_{q} x\right\rangle=\left\|A_{q} x\right\|^{2} .
\end{aligned}
$$

But $\left(A_{q}\right)^{*}=A^{*}-q^{\sigma} I$. Hence $q \in \sigma_{P}(A)$ if and only if $q^{\sigma} \in \sigma_{P}\left(A^{*}\right)$. Combining this with the result of Lemma 2 gives $\sigma_{P}(A)=\Gamma(A)$. Hence $\sigma_{R}(A)$ is empty.

COROLlaRY. If $A$ is a continuous normal real-linear operator, then $\sigma(A)=\Pi(A) \subseteq[W(A)]^{-}$.

\section{Functional calculus}

In this section some basic properties of the functional calculus for a sesquihermitian operator are investigated.

Given a quaternionic Hilbert space $\mathscr{H}$, let $\mathscr{L}_{+}(\mathscr{H})$ denote the real-linear space of Hermitian linear operators on $\mathscr{H}$ and $\mathscr{S}(\mathscr{H})$ the quaternion-linear space of sesquihermitian operators on $\mathscr{H}$. Then it follows from a result of Anderson [(1969), Theorem 2.9(c)] that the mapping $(A, f) \mapsto T(A) f$ is a 
continuous mapping from $\mathscr{S}(\mathscr{H}) \times C^{\infty}\left(\boldsymbol{R}^{4}\right)$ to $\mathscr{L}_{+}(\mathscr{H})$ with respect to the norm topology on $\mathscr{L}_{+}(\mathscr{H})$, the $C^{\infty}$-topology on $C^{\infty}\left(\boldsymbol{R}^{4}\right)$, and the topology on $\mathscr{S}(\mathscr{H})$ defined by the norm \|\|$_{s}$, where for a sesquihermitian operator $A=\sum_{\nu=0}^{3} i_{\nu} A_{\nu}$, we define $\|A\|_{S}=\Sigma_{\nu=0}^{3}\left\|A_{\nu}\right\|$. In general, \|\|$_{S}$ will depend on the choice of quaternion basis $\left(i_{1}, i_{2}, i_{3}\right)$. However, \|\|$_{s}$ is equivalent to the operator norm \|\| , as we shall show.

THEOREM 8. Let $A$ be a sesquihermitian operator on $\mathscr{H}$ with canonical decomposition $\sum_{\nu=0}^{3} i_{\nu} A_{\nu}$. Then $\|A\| \leqq\|A\|_{s} \leqq 4\|A\|$.

Proof. Clearly $\|A\| \leqq \Sigma_{\nu=0}^{3}\left\|i_{v} A_{\nu}\right\|=\Sigma_{\nu=0}^{3}\left\|A_{\nu}\right\|=\|A\| s$. Using the canonical decomposition formulae [5, Theorem 2] to find the $A_{\nu}$, we have for example:

$$
4 A_{0}=A-i_{1} A \cdot i_{1} I-i_{2} A \cdot i_{2} I-i_{3} A \cdot i_{3} I .
$$

So $\left\|A_{0}\right\| \leqq \frac{1}{4}\left(\|A\|+\left\|i_{1} A \cdot i_{1} I\right\|+\left\|i_{2} A \cdot i_{2} I\right\|+\left\|i_{3} A \cdot i_{3} I\right\|\right)=\|A\|$. Similarly $\left\|A_{\nu}\right\| \leqq\|A\|(\nu=1,2,3)$. Hence $\|A\|_{S} \leqq 4\|A\|$.

Corollary. The mapping $(A, f) \mapsto T(A) f$ is continuous with respect to the norm topology on $\mathscr{L}_{+}(\mathscr{H})$, the $C^{\infty}$-topology on $C^{\infty}\left(\boldsymbol{R}^{4}\right)$, and the uniformnorm topology on $\mathscr{P}(\mathscr{H})$.

Now let $C_{Q}^{\infty}\left(R^{4}\right)$ denote the quaternion-linear space of $C^{\infty}$-functions $f: \mathscr{Q} \rightarrow \mathscr{Q}$ and let $A$ denote a fixed sesquihermitian operator. Then the continuous real-linear mapping $T(A): C^{\infty}\left(\boldsymbol{R}^{4}\right) \rightarrow \mathscr{L}_{+}(\mathscr{H})$ can be extended to $C_{Q}^{\infty}\left(\boldsymbol{R}^{4}\right)$ by writing, for each $f \in C_{Q}^{\infty}\left(\boldsymbol{R}^{4}\right)$

$$
T(A) f=\sum_{\nu=0}^{3} i_{\nu} T(A) f_{\nu}
$$

where the real-valued functions $f_{v}$ are given by (1). Since each $T(A) f_{\nu}$ is a Hermitian linear operator, the operator $T(A) f$ is sesquihermitian.

THEOREM 9. For any fixed sesquihermitian operator $A$ on $\mathscr{H}, T(A)$ is a continuous (quaternion-) linear mapping from $C_{Q}^{\infty}\left(\boldsymbol{R}^{\star}\right)$ to $\mathscr{S}(\mathscr{H})$.

Proof. $\quad T(A)$ is clearly continuous and real-linear. Let $f \in C_{Q}^{\infty}\left(R^{\triangleleft}\right)$. Then for each $q \in \mathscr{Q}$ :

$$
i_{1} f(q)=-f_{1}(q)+i_{1} f_{0}(q)-i_{2} f_{3}(q)+i_{3} f_{2}(q) .
$$

So $\quad T(A)\left(i_{1} f\right)=-T(A) f+i_{1} T(A) f_{0}-i_{2} T(A) f_{3}+i_{3} T(A) f_{2}=i_{1} T(A) f$. Similarly for $i_{2}, i_{3}$, and hence for any quaternion $q$. Thus $T(A)$ is linear.

The space $C_{\mathbb{Q}}^{\infty}\left(\boldsymbol{R}^{4}\right)$ is actually an algebra over $\mathscr{2}$ with respect to pointwise multiplication of functions and $\mathscr{P}(\mathscr{H})$ is an algebra over $\mathscr{Q}$ with respect to composition of operators. However, $T(A): C_{Q}^{\infty}\left(R^{4}\right) \rightarrow \mathscr{S}(\mathscr{H})$ is not in general an algebraic homomorphism. 
For example, let $f$ be the identity function on 2 , so that $T(A) f=A$.

But

$$
\begin{aligned}
f^{2}(q)= & q^{2}=q_{0}^{2}-q_{1}^{2}-q_{2}^{2}-q_{3}^{2}+2 i_{1} q_{0} q_{1} \\
& +2 i_{2} q_{0} q_{2}+2 i_{3} q_{0} q_{3} \text { for each } q \in \mathscr{2} .
\end{aligned}
$$

Hence, using the fact that $T(A)$ acts "naturally" on the polynomials on $R^{4}$ [Anderson (1969), Theorems 2.4(c), 2.8]:

$$
\begin{aligned}
T(A)\left(f^{2}\right)= & A_{0}{ }^{2}-A_{1}{ }^{2}-A_{2}{ }^{2}-A_{3}{ }^{2}+i_{1}\left(A_{0} A_{1}+A_{1} A_{0}\right) \\
& +i_{2}\left(A_{0} A_{2}+A_{2} A_{0}\right)+i_{3}\left(A_{0} A_{3}+A_{3} A_{0}\right)
\end{aligned}
$$

which does not equal $A^{2}$ unless $A_{1}, A_{2}$, and $A_{3}$ commute with each other.

However, Anderson (1967) shows that the functional calculus for an $n$-tuple of commuting operators reduces to the orthodox one based on the commuting spectral measures.

DEFINITION. A sesquihermitian operator $A=\Sigma_{v=0}^{3} i_{v} A_{\nu}$ is called supernormal if the operators $A_{0}, A_{2}, A_{2}, A_{3}$ are mutually commuting.

This concept does not depend on the choice of quaternion basis. For if $A=\Sigma_{v=0}^{3} i_{v} A_{\nu}=\Sigma_{v=0}^{3} i_{\nu}{ }^{\prime} A_{\nu}{ }^{\prime}$ then [Powers (1973), Theorem 3] the $A_{\nu}{ }^{\prime}$ are real-linear combinations of the $A_{\nu}$, so that the $A_{\nu}{ }^{\prime}$ are mutually commuting if and only if the $A_{v}$ are.

THEOREM 10. If $A$ is a supernormal operator on $\mathscr{H}$, then the mapping $T(A): C_{Q}^{\infty}\left(\boldsymbol{R}^{4}\right) \rightarrow \mathscr{S}(\mathscr{H})$ is an algebraic homomorphism.

Proof. Suppose that $A$ is supernormal, so that the Hermitian linear operators $A_{0}, A_{1}, A_{2}, A_{3}$ are mutually commuting. We have to show that $T(A)$ is multiplicative. Let $f, g \in C_{Q}^{\infty}\left(R^{4}\right)$. Since $T(A)$ is linear, we can assume without loss of generality that $f, g \in C^{\infty}\left(\boldsymbol{R}^{4}\right)$-i.e., that $f$ and $g$ are real-valued.

Since the operators $A_{\nu}$ are Hermitian and linear, their spectral measures $E_{\nu}$ are linear. It can be deduced from complex Hilbert space spectral theory that the $E_{v}$ are mutually commuting, and we can define

$$
f\left(A_{0}, A_{1}, A_{2}, A_{3}\right)=\int_{\mathbf{R}^{4}} f\left(q_{0}, q_{1}, q_{2}, q_{3}\right) E_{0}\left(d q_{0}\right) \cdots E_{3}\left(d q_{3}\right)
$$

and similarly for $g$. Then according to Riesz and Sz.-Nagy (1965), § 111

$$
f\left(A_{0}, A_{2}, A_{3}\right) g\left(A_{0}, A_{1}, A_{2}, A_{3}\right)=(f g)\left(A_{0}, A_{1}, A_{2}, A_{3}\right) .
$$

But Anderson [(1967), §1.4] shows that:

$$
f\left(A_{0}, A_{1}, A_{2}, A_{3}\right)=T(A) f \text { for any } f \in C^{\infty}\left(R^{4}\right) .
$$

Hence $[T(A) f][T(A) g]=T(A)(f g)$. 
Thus the Weyl functional calculus for $C^{\infty}$-functions of a supernormal operator reduces to the classical one for several commuting operators, and is "natural" in its treatment of quaternion polynomials.

\section{References}

R. F. V. Anderson (1967), A functional calculus for non-commutative self-adjoint operators, Ph. D. Dissertation, Princeton University, 1967.

R. F. V. Anderson (1969), 'The Weyl functional calculus', J. Functional Analysis 4, 240-267.

R. F. V. Anderson (1970), 'On the Weyl functional calculus', J. Functional Analysis 6, 110-115.

P. R. Halmos (1967), A Hilbert Space Problem Book (Van Nostrand, Princeton, N. J., 1967).

N. C. Powers (1973), 'Real-linear operators on quaternionic Hilbert Space', Proc. Amer. Math. Soc. 40, $1-8$.

F. Riesz and B. Sz.-Nagy (1965), Functional Analysis (Ungar, New York, N. Y., 1965).

Department of Mathematics

University of Witwatersrand

Johannesburg

South Africa. 